\title{
Functional renormalization for repulsive Bose-Bose mixtures at zero temperature
}

\author{
Felipe Isaule, Ivan Morera, Artur Polls, and Bruno Juliá-Díaz \\ Departament de Física Quàntica i Astrofísica, Facultat de Física, and Institut de Ciències del Cosmos (ICCUB), \\ Universitat de Barcelona, Martí $i$ Franquès 1, E-08028 Barcelona, Spain
}

(Dated: November 3, 2020)

\begin{abstract}
We study weakly-repulsive Bose-Bose mixtures in two and three dimensions at zero temperature using the functional renormalization group (FRG). We examine the RG flows and the role of density and spin fluctuations. We study the condition for phase separation and find that this occurs at the mean-field point within the range of parameters explored. Finally, we examine the energy per particle and condensation depletion. We obtain that our FRG calculations compare favorably with known results from perturbative approaches for macroscopic properties.
\end{abstract}

\section{INTRODUCTION}

Weakly-interacting Bose gases have been subject of study for many decades [1, 2]. However, the interest on such gases greatly increased since the experimental realization of Bose-Einstein condensation with cold alkali atoms 3 - 5].

Experimentalists have been able to produce bosonic cold-atom gases in a variety of configurations and explore a range of interaction parameters and temperatures [6]. Theoretically, even though mean-field (MF) theory is able to give a qualitative description of Bose gases at low temperatures [7], for an accurate description, the effect of fluctuations needs to be included. For onecomponent Bose gases, the leading quantum correction in three dimensions was first calculated by Lee, Huang, and Yang (LHY) [8, 9]. Since then, further improvements have been provided with a variety of approaches [10]. In one and two dimensions, the effect of fluctuations is enhanced [11. Thus, corrections beyond the zero-point level in perturbation theory are more important, often requiring more careful treatments 12 14. In general, nowadays, one-component Bose gases are considered well described, and therefore the interest has shifted towards more sophisticated related systems.

Bose-Bose mixtures, gases with two species of bosons [2], have attracted attention in recent years. The interplay between the two components of the gas leads to rich physics such as the superfluid drag [15 17, Josephson effect [18, 19] and, depending if the inter-species interaction is repulsive or attractive, phase separation $[20$ 22 and self-bound droplets 23, 24]. Mixtures of bosons in cold atom gases were rapidly achieved experimentally by using bosonic atoms with two hyperfine states [2529], and there is currently a great effort on producing droplet phases in different configurations 30 33. Theoretically, Bose-Bose mixtures have been studied with different techniques, most noticeable perturbative approaches [34 39], Beliaev theory [40] and Monte Carlo (MC) simulations [24, 41, 42].

One alternative theoretical approach used to study quantum gases is the functional renormalization group (FRG) based on the effective average action [43, 44]. This is a non-perturbative technique where the full ef- fective action of the system is calculated by integrating out fluctuations, both quantum and thermal, by means of an RG equation. The FRG has been used with great success to study weakly-interacting one-component Bose gases in different dimensions [45 53]. One advantage of this approach is that the intricate long-distance physics is gradually incorporated during the RG flow [54]. Therefore the FRG does not suffer from the infrared (IR) divergences that plague perturbation theory [55, 56]. Furthermore, as a non-perturbative technique, the FRG has proved to deal with strongly-interacting systems with ease. For Bose gases, this has been relevant to study strongly-correlated superfluids in optical lattices [57].

Building upon the works on one-component gases, we study balanced and weakly-repulsive Bose-Bose mixtures at zero temperature within the FRG . We focus on both the RG flows and thermodynamics in two and three dimensions. We also examine the condition for phase separation. These configurations have been recently studied in detail using perturbative approaches in Refs. 36 39. RG approaches have also been used to study Bose-Bose mixtures. However, such studies have focused exclusively on the phase separation around the zero-density critical point [58, 59] and the superfluid phase transition [60, 61].

This work is organized in the following way. First, in Sec. II. we present the microscopic model within the path-integral formalism. In Sec. III, we present the FRG formalism and the ansatz for the effective action, including a short discussion on the physical inputs and the momentum regimes. The results are presented in Sec. IV] where we examine some features of the RG flows, the phase separation point, and finally, some thermodynamic properties.

\section{MICROSCOPIC MODEL}

We consider a non-relativistic gas of two species of bosons, $A$ and $B$, interacting through weak repulsive inter- and intra-species interactions. We work at length scales where the microscopic details of the interactions are not important, which are therefore represented by effective contact potentials with strength $g_{a b}$. We study the balanced mixture, that is, both species of bosons have equal masses $m=m_{A}=m_{B}$, equal chemical poten- 
tials $\mu=\mu_{A}=\mu_{B}$ and equal intra-species interactions $g=g_{A A}=g_{B B}$.

In a path-integral formulation 62] such gas is described by the Euclidean microscopic action

$$
\begin{aligned}
\mathcal{S}[\boldsymbol{\varphi}]=\int_{x} \sum_{a=A, B}\left[\psi _ { a } ^ { \dagger } \left(\partial_{\tau}\right.\right. & \left.-\frac{\nabla^{2}}{2 m}-\mu\right) \psi_{a} \\
& \left.+\sum_{b=A, B} \frac{g_{a b}}{2}\left|\psi_{a}\right|^{2}\left|\psi_{b}\right|^{2}\right]
\end{aligned}
$$

where $\int_{x}=\int_{0}^{\beta} \mathrm{d} \tau \int \mathrm{d}^{d} \mathbf{x}$, with $\tau=i t$ the imaginary time and $\beta=T^{-1}$ the inverse temperature. In this work we study the zero-temperature gas, hence $\beta \rightarrow \infty$. The action is a functional of the fields $\boldsymbol{\varphi}=\left(\psi_{A}, \psi_{A}^{\dagger}, \psi_{B}, \psi_{B}^{\dagger}\right)$, which represent the two bosonic species. Note that we set $\hbar=k_{B}=1$.

Throughout this article, we work in Fourier space $q=$ $(\omega, \mathbf{q})$. The kinetic terms in the action take the form

$$
\mathcal{S}_{\mathrm{kin}}[\boldsymbol{\varphi}]=\int_{q} \sum_{a=A, B}\left[\psi_{a}^{\dagger}\left(i \omega+\frac{\mathbf{q}^{2}}{2 m}\right) \psi_{a}\right]
$$

where the integral over $q$ at zero temperature is

$$
\int_{q}=\int_{-\infty}^{\infty} \frac{\mathrm{d} \omega}{2 \pi} \int \frac{\mathrm{d}^{d} \mathbf{q}}{(2 \pi)^{d}}
$$

The microscopic action defines the grand-canonical partition function

$$
\mathcal{Z}[\varphi]=\int D \varphi e^{-\mathcal{S}[\varphi]}
$$

which is a path integral over all configurations of the fields. The associated thermodynamic potential is obtained through

$$
\Omega=-\beta^{-1} \log \mathcal{Z}
$$

from which we can extract all the thermodynamic properties of the system. In the balanced mixture, its differential at zero temperature is

$$
\mathrm{d} \Omega=-P \mathrm{~d} \mathcal{V}_{d}-\langle N\rangle \mathrm{d} \mu
$$

where $\mathcal{V}_{d}$ is the $d$-dimensional volume, $P$ the pressure and $N$ the total number of particles. The energy density of the ground state is

$$
\frac{E}{\mathcal{V}_{d}}=-P+n \mu
$$

where $n=n_{A}+n_{B}$ is the total atom density. The energy per particle is obtained from $E / N=\left(E / \mathcal{V}_{d}\right) / n$.

\section{FUNCTIONAL RENORMALIZATION GROUP}

The strategy of the FRG is to generate the effective action $\Gamma$ of a system by smoothly taking fluctuations into account with an RG group equation [43, 44]. To achieve this, one considers a regulator function $\mathbf{R}(q ; k)$, which suppresses all fluctuations for momenta $q \lesssim k$. This regulator is added to the theory as a mass term

$$
\Delta \mathcal{S}_{k}[\boldsymbol{\varphi}]=\int_{q} \boldsymbol{\varphi}^{\dagger}(q) \mathbf{R}_{k}(q) \boldsymbol{\varphi}(q)
$$

so the grand-canonical partition function becomes $k$ dependent

$$
\mathcal{Z}_{k}[\mathbf{J}]=\int D \varphi e^{-\mathcal{S}[\boldsymbol{\varphi}]-\Delta \mathcal{S}_{k}[\boldsymbol{\varphi}]+\int_{x} \mathbf{J} \cdot \boldsymbol{\varphi}},
$$

where we also added source fields $\mathbf{J}$. The $k$-dependent effective action is defined by means of a Legendre transformation

$$
\Gamma_{k}[\boldsymbol{\phi}]=-\log \mathcal{Z}_{k}[\mathbf{J}]+\int_{x} \mathbf{J} \cdot \boldsymbol{\phi}-\Delta \mathcal{S}_{k}[\boldsymbol{\phi}],
$$

where $\phi(x)=\langle\boldsymbol{\varphi}(x)\rangle$ are classical fields. At a UV scale $k=\Lambda$, all fluctuations are suppressed and the effective action is simply the microscopic action; $\Gamma_{\Lambda}[\phi]=\mathcal{S}[\phi]$. In contrast, for $k \rightarrow 0$, all fluctuations have been taken into account and $\Gamma_{0}[\phi]$ is the physical effective action. $\Gamma_{0}$ is the generator of the one-particle irreducible Green's functions, from which we can extract the physical properties of interest. At equilibrium

$$
\left.\frac{\delta \Gamma}{\delta \phi}\right|_{\phi_{0}}=0
$$

the effective action is related to the thermodynamic potential $\Omega$ through

$$
\Omega=\Gamma\left[\phi_{0}\right] / \beta
$$

which enables us to extract the thermodynamic properties of the gas.

The flow of $\Gamma_{k}$ as a function of $k$ is dictated by the Wetterich equation 43 .

$$
\partial_{k} \Gamma_{k}=\frac{1}{2} \operatorname{tr}\left[\partial_{k} \mathbf{R}_{k}\left(\boldsymbol{\Gamma}_{k}^{(2)}+\mathbf{R}_{k}\right)^{-1}\right],
$$

where $\boldsymbol{\Gamma}_{k}^{(2)}$ is the matrix with the second-functional derivatives of $\Gamma_{k}$. The trace denotes both a trace and an integral over internal momentum $q$.

In most applications, the Wetterich equation cannot be solved directly, and thus approximations need to be employed. In this work, we propose an ansatz for the effective action $\Gamma_{k}$ based on a derivative expansion (DE) truncated to a small number of $k$-dependent terms [44. Within this approximation, the Wetterich equation becomes a set of coupled differential equations that can be 
solved numerically using standard methods. The DE is generally appropriate to study the long-distance physics and thermodynamic properties as in this work.

In the following we present the ansatz for the effective action and details of the flow equations. For detailed reviews on the FRG framework we refer to Refs. [44, 6366 .

\section{A. Effective action}

We use an ansatz and strategy analogous to those used for one-component Bose gases [45,47]. Our ansatz reads

$$
\begin{array}{r}
\Gamma_{k}[\phi]=\int_{x}\left[\sum_{a=A, B} \psi_{a}^{\dagger}\left(S \partial_{\tau}-\frac{Z}{2 m} \nabla^{2}-V \partial_{\tau}^{2}\right) \psi_{a}\right. \\
\left.+U\left(\rho_{A}, \rho_{B} ; \mu\right)\right]
\end{array}
$$

where $\rho_{a}=\phi_{a}^{\dagger} \phi_{a}$ and $S, Z$ and $V$ are $k$-dependent renormalization factors, which we consider as fieldindependent. The effective potential $U$ is expanded around the $k$-dependent order parameters $\rho_{a, 0}=\left\langle\phi_{a}^{\dagger} \phi_{a}\right\rangle$, which are equal for the balanced mixture $\rho_{0}=\rho_{A, 0}=$ $\rho_{B, 0}$. We are interested in the phase where the $U(1)$ symmetry of each species is broken, thus $\rho_{0}>0$ for all $k$ [47. In this phase, the effective potential truncated up to quartic order in the fields reads

$$
\begin{aligned}
U\left(\rho_{A}, \rho_{B} ; \mu\right)= & -\sum_{a=A, B}\left(n_{0}+n_{1}\left(\rho_{a}-\rho_{0}\right)\right)(\tilde{\mu}-\mu) \\
& +\sum_{a, b=A, B} \frac{\lambda_{a b}}{2}\left(\rho_{a}-\rho_{0}\right)\left(\rho_{b}-\rho_{0}\right),
\end{aligned}
$$

where $\lambda_{A A}=\lambda_{B B}=\lambda$. We have added shifts $\tilde{\mu}$ from the from the physical chemical potential $\mu$, so we can follow the flow of the densities $n_{0}=n / 2$ of each species of bosons (see Ref. [47] for details). The condensate densities are given by $\rho_{0}$, whereas the superfluid densities are given by the phase stiffness $\rho_{s}=Z \rho_{0}$ [50, 67]. Thus, the physical condensate and superfluid fractions are obtained from the values at $k=0$ of $\Omega_{s}=\rho_{s} / n_{0}$ and $\Omega_{c}=\rho_{0} / n_{0}$, respectively.

When the $U(1)$-symmetry is broken, it is useful to decompose the complex boson fields $\psi_{a}$ into orthogonal real fields

$$
\psi_{a}(x)=\frac{1}{\sqrt{2}}\left(\psi_{a, 1}(x)+i \psi_{a, 2}(x)\right) .
$$

In this work we fix both order parameters $\rho_{A, 0}$ and $\rho_{B, 0}$ at the same direction. Thus, we evaluate the fields at $\left\langle\psi_{a, 1}\right\rangle=\left(2 \rho_{0}\right)^{1 / 2}$ and $\left\langle\psi_{a, 2}\right\rangle=0$. The inverse propagator evaluated at this point reads

$$
\mathbf{G}_{k}^{-1}(q)=\left(\begin{array}{cc}
\mathbf{G}_{k, \psi}^{-1}(q) & \boldsymbol{\Sigma}_{k, A B} \\
\boldsymbol{\Sigma}_{k, B A} & \mathbf{G}_{k, \psi}^{-1}(q)
\end{array}\right)
$$

where

$$
\begin{gathered}
\mathbf{G}_{k, \psi}^{-1}(q)=\left(\begin{array}{cc}
E_{1, k}(\mathbf{q})+V \omega & S \omega \\
-S \omega & E_{2, k}(\mathbf{q})+V \omega
\end{array}\right) \\
\boldsymbol{\Sigma}_{k, A B}=\boldsymbol{\Sigma}_{k, B A}=\left(\begin{array}{cc}
2 \lambda_{A B} \rho_{0} & 0 \\
0 & 0
\end{array}\right)
\end{gathered}
$$

and

$$
\begin{aligned}
& E_{1, k}(\mathbf{q})=Z \frac{\mathbf{q}^{2}}{2 m}+2 \lambda \rho_{0}+n_{1}(\tilde{\mu}-\mu)+R_{k}(\mathbf{q}), \\
& E_{2, k}(\mathbf{q})=Z \frac{\mathbf{q}^{2}}{2 m}+n_{1}(\tilde{\mu}-\mu)+R_{k}(\mathbf{q}) .
\end{aligned}
$$

As with one-component gases, $\psi_{a, 1}$ represent fluctuations of the longitudinal modes, whereas $\psi_{a, 2}$ represent fluctuations of the massless Goldstone modes [46].

In this work we use the optimized Litim regulator [68],

$$
R_{k}(\mathbf{q})=\frac{Z}{2 m}\left(k^{2}-\mathbf{q}^{2}\right) \Theta\left(\mathbf{q}^{2}-k^{2}\right),
$$

where $\Theta$ is the Heaviside step function. This regulator allows us to perform the momentum integrals analytically before solving the differential equations. Although this regulator is frequency-independent, it has proven to give reasonable results for quantum gases [49, 65.

The flow equations for the $k$-dependent are obtained from the appropriate projections of the Wetterich equation (13) into the ansatz 14, which are analogous to those for one-component gases [47, 53]. These can be found in App. A.

We stress that in our ansatz, we do not include the effect of the relative phase between the two condensates, and therefore we do not describe the non-dissipative drag between superfluids 15, 16. Within our truncation, the interaction between both species of bosons is driven exclusively by the coupling $\lambda_{A B}$, which only couples the phase-independent terms $\rho_{a}$. However, because phase fluctuations are not too strong in two and three dimensions at zero temperature 69, the superfluid drag does not considerably affect their macroscopic properties. For details on the effect of the superfluid drag in one dimension see Ref. [70].

\section{B. Initial conditions and physical inputs}

The flow is started at a UV scale $\Lambda$ much larger than the physical scale set by the chemical potential. We obtain the initial conditions of the RG flow by imposing that $\Gamma_{\Lambda}=\mathcal{S}$. For the homogeneous (miscible) phase, these are

$$
\begin{gathered}
S(\Lambda)=Z(\Lambda)=1, \quad V(\Lambda)=0 \\
\rho_{0}(\Lambda)=n_{0}(\Lambda)=\frac{\mu}{\lambda(\Lambda)+\lambda_{A B}(\Lambda)}, \quad n_{1}(\Lambda)=1,
\end{gathered}
$$

where $\mu>0$. The interaction terms $\lambda$ and $\lambda_{A B}$ need to be renormalized so they can be connected to the $s$-wave 
scattering lengths $a$ and $a_{A B}$ associated to the intra- and inter-species interactions, respectively. With this, the initial conditions are completely defined in terms of the physical inputs $\mu, a$ and $a_{A B}$. We impose that the interaction terms at $k=0$ in vacuum are equal to the known two-body $T$-matrices: $\lambda(0)=T^{2 B}, \lambda_{A B}(0)=T_{A B}^{2 B}[47$. In two and three dimensions these are 62

$$
T_{\alpha}^{2 B}=\left\{\begin{array}{ll}
\frac{4 \pi / m}{\log \left(-2 / m|\mu| a_{\alpha}^{2}\right)-2 \gamma_{E}} & : d=2 \\
\frac{4 \pi a_{\alpha}}{m} & : d=3
\end{array},\right.
$$

where $T_{\alpha}^{2 B}=T^{2 \mathrm{~B}}, T_{A B}^{2 \mathrm{~B}}, a_{\alpha}=a, a_{A B}$ and $\gamma_{E} \approx 0.577$ is the Euler-Mascheroni constant. For the optimized regulator 22 we obtain the following initial conditions [47, 53]

$$
\lambda_{\alpha}(\Lambda)=\left\{\begin{array}{ll}
\frac{4 \pi / m}{1-2 \gamma_{E}-\log \left(a_{\alpha}^{2} \Lambda^{2} / 4\right)} & : d=2 \\
\frac{4 \pi / m}{a_{\alpha}^{-1}-4 \Lambda / 3 \pi} & : d=3
\end{array},\right.
$$

where $\lambda_{\alpha}=\lambda, \lambda_{A B}$. Note that although we have to choose a high starting scale $\Lambda$, for repulsive interactions we must choose $\Lambda \lesssim a_{\alpha}^{-1}$, otherwise $\lambda_{\alpha}(\Lambda)<0$ (for details see Ref. [53]).

Furthermore, we must also choose $a_{A B}<a$ so that the spin mass term $2 \rho_{0}\left(\lambda-\lambda_{A B}\right)>0$ at the starting scale $\Lambda$ (see next subsection). A stronger inter-species repulsion corresponds to a flow that starts in the separable (nonmiscible) phase 2, which is not described by our ansatz. In subsection IVB we show that the FRG flow signals the physical separable phase by the vanishing of the spin healing scale 18 .

\section{Scale regimes}

As the momentum scale $k$ is lowered, the RG flow gradually incorporates into $\Gamma_{k}$ the fluctuations associated with different scales. Therefore, we can identify different scale regimes depending on the relevant physics.

In a one-component Bose gas with broken $U(1)$ symmetry, the flow can be separated into a Gaussian regime (for $Z k^{2} / 2 m \ll 2 \lambda \rho_{0}$ ) where both longitudinal and Goldstone fluctuations play a similar role, and a Goldstone regime (for $Z k^{2} / 2 m \ll 2 \lambda \rho_{0}$ ) where the flow is dominated by Goldstone fluctuations (for details see Ref. 46]). In order to identify the analogous regimes in a Bose-Bose mixture, we examine the behavior of the determinant of $\mathbf{G}_{k}^{-1}(17)$. Within our truncation, it reads

$$
\begin{gathered}
\operatorname{det}\left(\mathbf{G}_{k}^{-1}\right)=\left(S^{2} \omega^{2}+\left(V \omega^{2}+E_{1, k}^{(+)}\right)\left(V \omega^{2}+E_{2, k}\right)\right) \\
\times\left(S^{2} \omega^{2}+\left(V \omega^{2}+E_{1, k}^{(-)}\right)\left(V \omega^{2}+E_{2, k}\right)\right),
\end{gathered}
$$

where

$$
\begin{aligned}
E_{1, k}^{( \pm)}(\mathbf{q}) & =E_{1, k}(\mathbf{q}) \pm_{1} 2 \lambda_{A B} \rho_{0} \\
& =Z \frac{\mathbf{q}^{2}}{2 m}+2\left(\lambda \pm \lambda_{A B}\right) \rho_{0}+R_{k}(\mathbf{q})
\end{aligned}
$$

and $E_{1, k}$ and $E_{2, k}$ are defined in Eqs. 20 and 21. Note that we take $\tilde{\mu}=\mu$.

The poles of the propagator and the dispersion relations are extracted from $\operatorname{det}\left(\mathbf{G}_{k}^{-1}\right)=0$ (see App. B. From Eq. 26 we see that there are two solution branches. The positive branch $\left(E_{1, k}^{(+)}\right)$corresponds to the density (in-phase) mode, whereas the negative branch $\left(E_{1, k}^{(-)}\right)$corresponds to the spin (out-of-phase) mode [36].

It is easy to see that the dependency on momentum in Eq. 26 depends on how the mass-like terms $2\left(\lambda \pm \lambda_{A B}\right) \rho_{0}$ compare to the kinetic terms. Thus, we can identify different scale regimes from the dimensionless quantities

$$
\omega_{h, \pm}=\frac{Z^{2} k^{2} / 2 m}{2\left(\lambda \pm \lambda_{A B}\right) \rho_{0}}
$$

which for $\lambda_{A B}=0$ recovers the analogous expression in one-component gases 46. We define the momentum healing scales $k_{h, \pm}$ from the points in the flow where $\omega_{h, \pm}=1$. Note that even though we define $k_{h, \pm}$ in analogy to the physical healing lengths [2], $k_{h, \pm}$ correspond to scales in the RG flow and are not extracted from the physical inputs.

Similarly to one-component gases, the Gaussian regime is defined for high scales $k \gg k_{h, \pm}\left(\omega_{h, \pm} \gg 1\right)$ where the mass-like terms $2\left(\lambda \pm \lambda_{A B}\right) \rho_{0}$ are small and $E_{1, k}^{( \pm)} \approx E_{2, k}$. At these high scales many-body effects are not important, and thus the flows are similar to those in vacuum [47]. On the opposite side, the Goldstone regime is defined for low scales $k \ll k_{h, \pm}\left(\omega_{h, \pm} \ll 1\right)$ where $E_{1, k}^{( \pm)} \approx 2\left(\lambda \pm \lambda_{A B}\right) \rho_{0}$ and Goldstone fluctuations become dominant.

Between these two regimes, at scales $k_{h,-} \lesssim k \lesssim k_{h,+}$, the mixture develops an additional regime where the effect of the interaction between both species of bosons becomes most important. In particular, around $k_{h,+}$ and $k_{h,-}$ fluctuations of the density and spin modes dominate, respectively. This is easy to see in the cases where $k_{h,-} \ll k_{h,+}$. Here, around $k_{h,+}$ the spin mode is in a Gaussian-like regime as $E_{1, k}^{(-)} \approx E_{2, k}$, whereas the density mass term $2\left(\lambda+\lambda_{A B}\right) \rho_{0}$ is of the order of the kinetic terms and density fluctuations become important. On the hand, around $k_{h,-}$ the density mode is in a Goldstone-like regime $E_{1, k}^{(+)} \approx 2\left(\lambda+\lambda_{A B}\right) \rho_{0}$ and spin fluctuations dominate.

We provide additional discussion in App. B where we examine the dispersion relations extracted from Eq. (26). 


\section{RESULTS}

In this section, we present flows of the interaction terms $\lambda$ and $\lambda_{A B}$. We analyze the scale regimes and the phase separation point. We also present results for macroscopic thermodynamic properties and compare with analytical results from perturbative approaches.

\section{A. Flow of the interactions}

The RG flows of the mixture have similar properties to those of one-component gases [45, 47], Therefore, here we focus on the new features. Details of the RG flows are given in App. C

The intra-species interaction coupling $\lambda$ maintains its one-component behavior for small $k$ in the Goldstone regime: $\lambda$ vanishes for $k \rightarrow 0$ linearly with $k$ in two dimensions and logarithmically in three dimensions [45]. This is required to satisfy the vanishing of the anomalous self-energy [54, 56]. Similarly, we observe that $\lambda_{A B}$ vanishes for $k \rightarrow 0$ as well. However, it is relevant to examine how its flow compares to that of $\lambda$.

Fig. 1 shows examples of flows of the ratio $\lambda_{A B} / \lambda$ in two and three dimensions for different values of $a_{A B} / a$ (for the individual flows see Fig. 7). Note that we use a different range of $a_{A B} / a$ in two and three dimensions because of the different dependence on the scattering length (see Eq. (24)). We observe that this ratio shows distinct features during the different regimes of the flow. At high scales the ratio increases as we lower $k$ until it reaches its $\mathrm{MF}$ value $\approx T^{2 B} / T_{A B}^{2 B}$ around $k_{h,+}$, showing the dependence on $a_{A B}$. On the other hand, for small scales $k \lesssim k_{h,-}$ the ratio decreases until it vanishes for $k \rightarrow 0$. We obtain that $\lambda_{A B} / \lambda$ vanishes for small scales linearly with $k$ in two dimensions and logarithmically in three dimensions.

Because $\lambda_{A B} / \lambda$ vanishes for small $k$, the density and spin branches in Eq. 26) are not separated for $k \rightarrow 0$ and both components of the mixture decouple at long distances. This may seem counterintuitive from a Bogoliubov perspective, where both modes are always present in a perturbative expansion. However, in our RG formalism, the fluctuations of density and spin modes are incorporated around their associated scales $k_{h,+}$ and $k_{h,-}$, and the effective action at long distances do not necessarily maintain the form of the microscopic theory.

It is also worth noting the behavior of $k_{h, \pm}$. The density healing scale $k_{h,+}$ is roughly independent of $a_{A B}$ for a fixed chemical potential. On the other hand, the spin healing scale $k_{h,-}$ depends strongly on the interspecies interaction, decreasing as $a_{A B}$ increases. Furthermore, we observe that $\lambda_{A B} / \lambda \approx T^{2 B} / T_{A B}^{2 B}$ for a larger range of scales as $k_{h,-}$ decreases, approaching the solution $\lambda_{A B} / \lambda \rightarrow 1$ for $k \rightarrow 0$ at the phase separation point $a_{A B}=a$. We examine this in the following.

\section{B. Healing scales and phase separation}

The condition for phase-separation during the RG flow corresponds to $\lambda=\lambda_{A B}$ [58]. Under this condition, from Eq. (28) we have that $\omega_{-} \rightarrow \infty$, and thus the spin healing scale vanishes $k_{h,-} \rightarrow 0$. In contrast, the density healing scale remains finite. The same condition applies within perturbative approaches, where the similarly defined momentum healing scales are defined by [35, 37]

$$
p_{h, \pm}^{2}=4 m \mu \frac{g \pm g_{A B}}{g+g_{A B}} .
$$

At the MF phase separation point $g_{A B}=g$, we have $p_{h,-}=0$ and $p_{h,+}>0$. Therefore, to study the phaseseparation point, we can examine the behavior of $k_{h,-}$ for different physical inputs.

Fig. 2 shows healing scales for a range of inter-species scattering lengths $a_{A B}$. We compare our FRG calculations with the Bogoliubov healing scales 29 , where we use the $T$-matrices defined in Eq. (24) as the interactions $g$ and $g_{A B}$.

As discussed in the last subsection, the density healing scale $k_{h,+}$ is roughly independent of $a_{A B} / a$, as expected for the balanced mixture. On the other hand, the spin healing scale $k_{h,-}$ decreases as $a_{A B}$ increases. We obtain that $k_{h,-}$ vanishes for $a_{A B} \rightarrow a$. Therefore, we obtain that the phase separation occurs for equal interand intra-species interactions $a_{A B}=a$, coinciding with the MF prediction. We can understand this from the behavior of $\lambda_{A B} / \lambda$ discussed in the last subsection. Because this ratio decreases for $k<k_{h,-}$, then the condition $\lambda_{A B}=\lambda$ is satisfied only for $a_{A B}=a$.

Our result contrasts with the phase-separation point obtained with RG methods in Ref. [58], where it is stated that in two dimensions, the phase separation occurs for $a_{A B}<a$ at logarithmically low densities. However, that work study the mixture around the zero-density critical point, and thus we might not be exploring that regime. An FRG study of the quantum phase transition of the mixture around the zero-density point can be done by extending the same analysis for one-component gases from Ref. [46]. However, this is beyond the scope of the current work.

\section{Thermodynamics}

In the following we present results for the energy per particle $E / N$ and the condensation depletion $\Delta \Omega_{c}=1-$ $\Omega_{c}$. $E / N$ is computed from the physical values of the pressure, density and chemical potential as follows from Eq. (7). For details on how to extract the pressure see Ref. 53. 


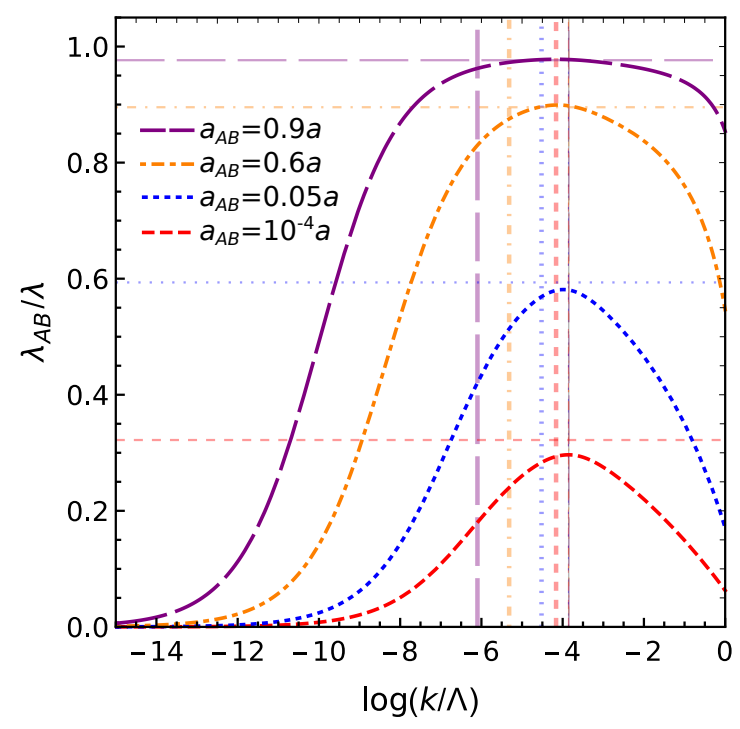

(a) Two dimensions

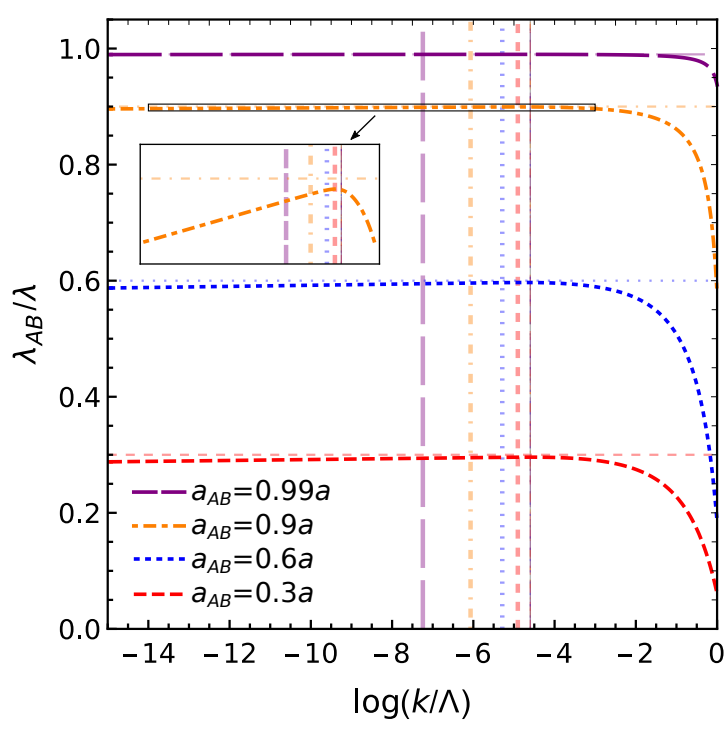

(b) Three dimensions

FIG. 1. Flows of the ratio $\lambda_{A B} / \lambda$ for different values of $a_{A B} / a$ as a function of $k$ in two and three dimensions for $m a^{2} \mu=10^{-4}$. The four thick vertical lines on the left denote $k_{h,-}$, whereas the thin vertical lines on the right (on top of each other) denote $k_{h,+}$. The horizontal lines correspond to the ratios $T_{A B}^{2 B} / T^{2 B}$, where these are defined in Eq. 24,. The inset in (b) enlarges one of the curves to show its logarithmic flow. The other curves show similar logarithmic behavior.

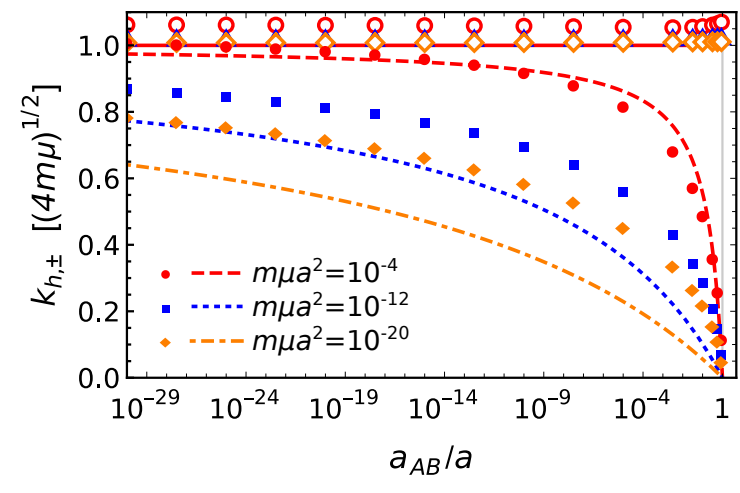

(a) Two dimensions

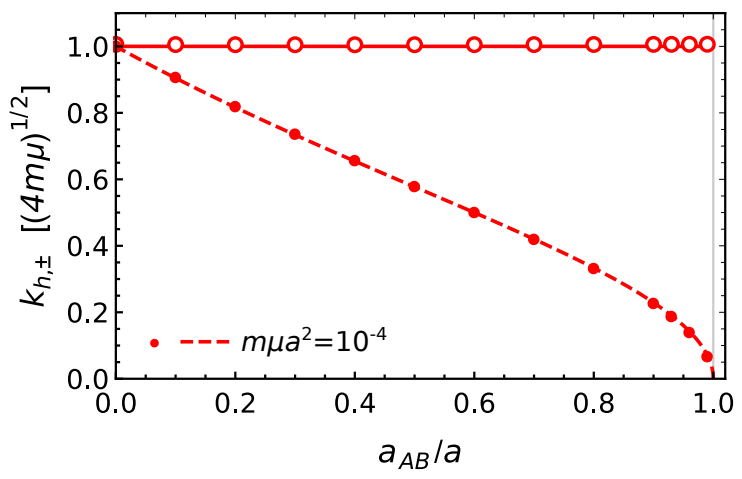

(b) Three dimensions

FIG. 2. Healing scales $k_{h, \pm}$ in two and three dimensions as a function of $a_{A B} / a$. Circles correspond to FRG results for $k_{h,+}$ (open) and $k_{h,-}$ (filled), whereas lines correspond to the Bogoliubov healing scales $p_{h, \pm}(29)$. In three dimensions the healing scale is independent of the chemical potential, and thus we plot one choice of $m \mu a^{2}$.

\section{Three dimensions}

Fig. 3 shows results for $E / N$ and $\Delta \Omega$ in three dimensions for a range of intra-species scattering lengths $a$ for different ratios of $a_{A B} / a$. Note that $a_{A B}=0$ corresponds to the one-component limit. We compare the energy per particle with the perturbative result [34]

$$
\frac{E}{N}=\frac{\pi n}{m}\left(a+a_{A B}\right)+\frac{32 \sqrt{2 \pi}}{15} \frac{n^{3 / 2} a^{5 / 2}}{m} f\left(a_{A B} / a\right),
$$

where $f(x)=(1+x)^{5 / 2}+(1-x)^{5 / 2}$ and $n=2 n_{0}$ is the total density. The first term corresponds to the MF so- lution and the second term to the LHY correction. Similarly, we compare the condensate depletion with the LHY result [1]

$$
\Delta \Omega_{c}=\frac{4}{3 \sqrt{2 \pi}}\left(n a^{2}\right)^{3 / 2} h\left(a_{A B} / a\right),
$$

where $h(x)=(1+x)^{3 / 2}+(1-x)^{3 / 2}$.

We obtain an excellent agreement with the LHY results, showing that our calculations correctly incorporate the effect of quantum fluctuations and of the inter-species interaction. We stress that the LHY corrections give an accurate description of Bose gases in three dimensions for this range of $n a^{3}$ [10. In particular, the accuracy 


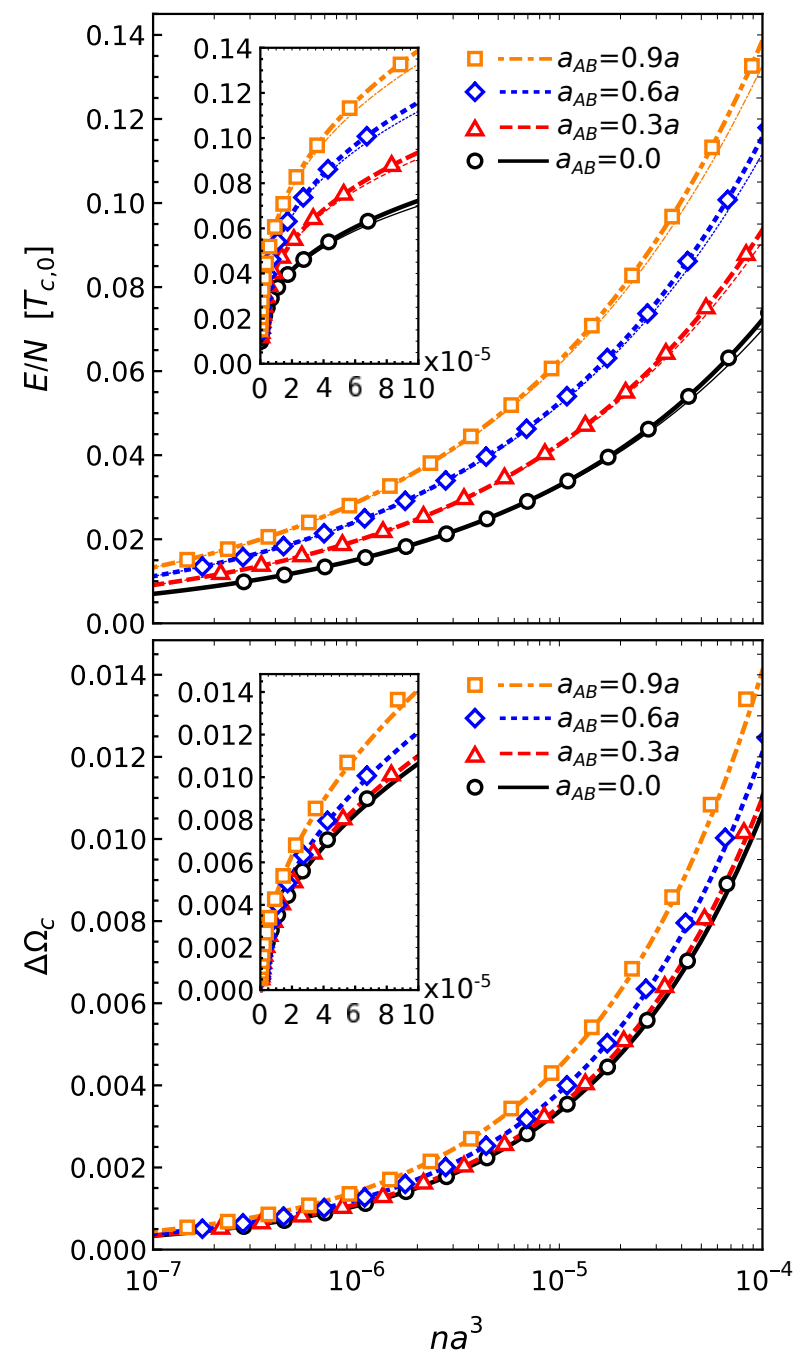

FIG. 3. Energy per particle $E / N$ and condensate depletion $\Delta \Omega_{c}$ in three dimensions as a function of the concentration parameter $n a^{3}$, where $n=2 n_{0}$ is the total density of atoms. $E / N$ is scaled in terms of the critical temperature of an ideal Bose gas $T_{c, 0}=\frac{2 \pi}{m}\left(n_{0} / \zeta(3 / 2)\right)^{2 / 3}$. Markers correspond to FRG results, thin lines to the MF solutions and thick lines to $\mathrm{MF}+\mathrm{LHY}$ solutions 30 and 31 . The insets show the results in a linear scale.

of the LHY result for the condensate depletion in onecomponent gases has been proved experimentally [72].

\section{Two dimensions}

Fig. 4 shows results for $E / N$ and $\Delta \Omega_{c}$ in two dimension. We compare the energy per particle with the perturbative result 38

$$
\begin{aligned}
\frac{E}{N}=\frac{\pi n}{m} \zeta_{+} & \left(n, a, a_{A B}\right)+\frac{\pi n}{2 m} \sum_{ \pm} \zeta_{ \pm}^{2}\left(n, a, a_{A B}\right) \\
& \times\left(2 \gamma_{E}+1 / 2+\log \left(\pi \zeta_{ \pm}\left(n, a, a_{A B}\right)\right)\right.
\end{aligned}
$$

where

$$
\zeta_{ \pm}\left(n, a, a_{A B}\right)=\frac{1}{\left|\log \left(n a^{2} / 2\right)\right|} \pm \frac{\Theta\left(a_{A B}\right)}{\left|\log \left(n a_{A B}^{2} / 2\right)\right|}
$$

As in three dimensions, the first term in Eq. (32) corresponds to the MF result and the second to the LHY-type correction. Additionally, we compare with MC results of $E / N$ for the one-component gas [73]. For the condensate depletion, we compare with the recently obtained expression for the one-component gas [74]

$$
\Delta \Omega_{c}=\frac{1}{\left|\log \left(n a^{2} / 2\right)\right|+\log \left(\left|\log \left(n a^{2} / 2\right)\right|\right)+C},
$$

where $C \approx-\log (\pi)-2 \gamma_{E}-2.63$.

For $E / N$, we obtain a reasonable agreement between our FRG results and the analytical expression. We stress that because of the enhanced effect of fluctuations in two dimensions, the LHY-type correction is not as accurate, and thus we expect that our results give a better description of this system. In particular, for the one-component limit $\left(a_{A B}=0\right)$, we obtain a slighter better agreement with the MC results, which we can consider exact. Also, note the larger effect of the LHY correction to the MF result compared to three dimensions, showing the enhanced effect of quantum fluctuations.

For $\Omega_{c}$ we obtain that in the one-component limit, our results are comparable to those of Eq. (34). However, we stress that FRG calculations with better truncations are needed in order to prove the robustness of our results. Most interesting is the effect of the inter-species interaction. At first glance it seems that the condensate depletion in two dimensions is not as sensitive to the inter-species interaction as in three dimensions. However, in our figures, $\Omega_{c}$ is one order of magnitude greater in two dimensions. The larger condensate depletion is caused by the enhanced fluctuations in two dimensions, which are at the critical dimension of destroying the condensate [75]. Therefore, in two dimensions, the depletion is mostly driven by the quantum fluctuations associated with the lower dimensionality, and the effects of the mixture become less noticeably.

\section{CONCLUSIONS}

In this work, we study two- and three-dimensional balanced Bose-Bose mixtures at zero temperature within the FRG. We generalize previous work on one-component gases to now consider two species of bosons interacting through weak and repulsive intra- and inter-species interactions.

We find that the scale-dependent inter-species interaction coupling vanishes at long distances. However, the RG flow correctly takes the inter-species interaction effect into account as fluctuations of density and spin modes are incorporated at their associated momentum scales. We also study the phase separation condition, which in our 


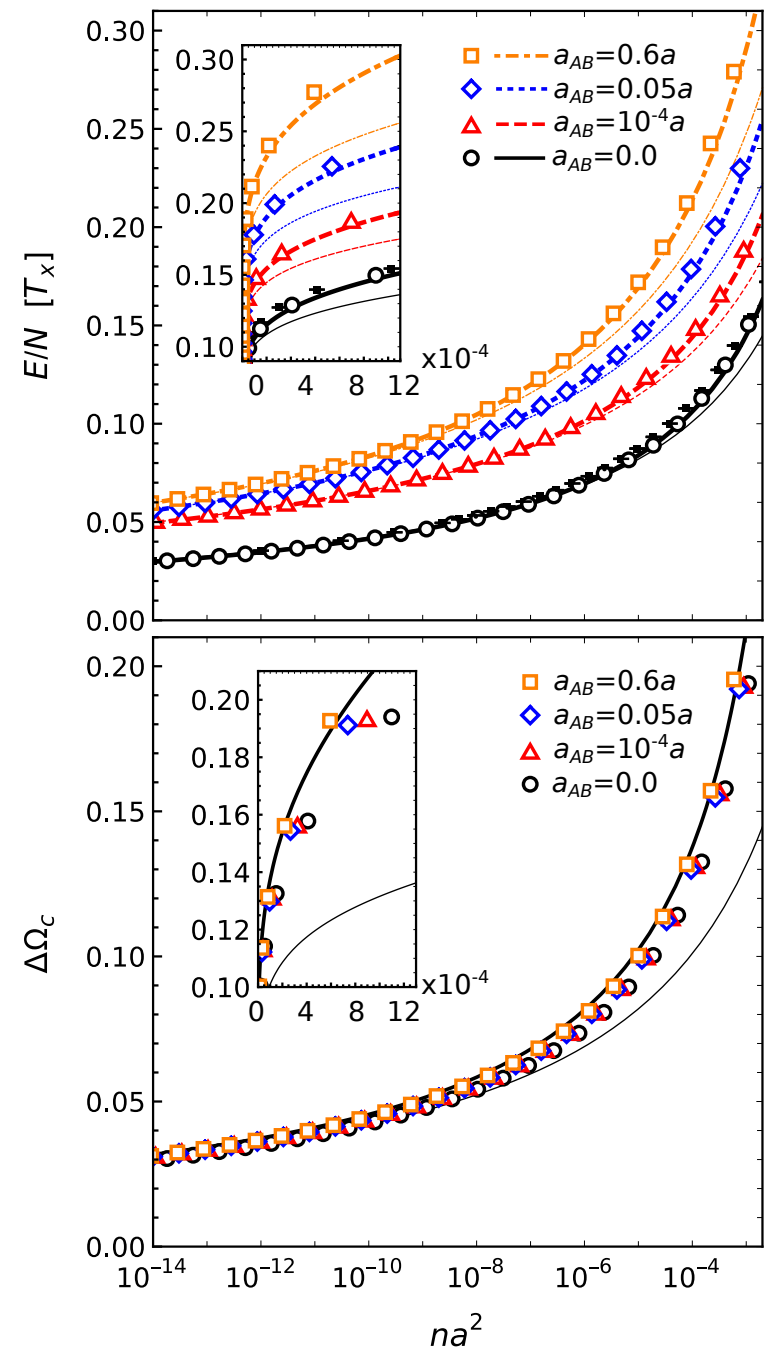

FIG. 4. Energy per particle $E / N$ and condensate depletion $\Delta \Omega_{c}$ in two dimensions as a function of the concentration parameter $n a^{2}$, where $n=2 n_{0}$ is the total density of atoms. $E / N$ is scaled in terms of the characteristic temperature $T_{x}=2 \pi n_{0} / m$. Markers correspond to FRG results. (Top): Thin lines correspond to the MF solution and thick lines to $\mathrm{MF}+\mathrm{LHY}$ solution (32), whereas the filled black circles correspond to MC simulations for the one-component gas from Ref. 73. (Bottom): Thin line corresponds to the analytical expression at leading order $\Delta \Omega_{c}=1 /\left|\log \left(n a^{2} / 2\right)\right|$ and the thick line to Eq. (34). The insets show the results in a linear scale.

formalism can be identified by the vanishing of the momentum spin healing scale. We find that in both two and three dimensions, the phase separation occurs at the MF point of equal scattering lengths $a_{A B}=a$. Finally, in order to examine macroscopic thermodynamic properties, we calculate the energy per particle and condensation depletion for a range of interaction parameters. We obtain good agreement with analytical results from perturbative approaches. We find some deviations from these results in two dimensions, as expected from the enhanced effect of fluctuations.

Having demonstrated that the FRG is capable of studying Bose-Bose mixture, we intend to explore in future work how to implement the interaction between the relative phases of the condensates within our framework. This is necessary to study the mixture at finite temperatures and the superfluid phase transition where the superfluid drag becomes important [17, 61]. We also intend to study the mixture around the quantum transition at zero density to check if the MF phase separation point changes as suggested in other works. Other relevant related extensions are studies of quantum mixtures in different configurations. Particularly interesting are Bose-Bose mixture with attractive inter-species interaction. These would enable us to explore the stronglyinteracting regime, which presents rich physics, including droplet phases and dimerization [76, 777. Because perturbative approaches are not suitable to study this regime, an FRG study could provide a more robust picture of these systems.

\section{ACKNOWLEDGMENTS}

This work has been partially supported by MINECO (Spain) Grant No.FIS2017-87534-P. We acknowledge financial support from Secretaria d'Universitats i Recercadel Departament d'Empresa i Coneixement de la Generalitat de Catalunya, co-funded by the European Union Regional Development Fund within the ERDF Operational Program of Catalunya (project QuantumCat, ref. 001-P-001644). We acknowledge helpful discussions with Michael C. Birse.

\section{Appendix A: Flow equations}

The flow of each coupling is driven by the appropriate projection of the Wetterich equation (13) into the ansatz (14). The flow of the order parameter is driven by the equilibrium condition $\delta \Gamma / \delta \psi_{a, 1}=0$, thus

$$
-\sqrt{2 \rho_{0}}\left(\lambda+\lambda_{A B}\right) \dot{\rho}_{0}=\left.\frac{\delta \dot{\Gamma}}{\delta \psi_{A, 1}}\right|_{\rho_{0}, \mu},
$$

where $\dot{f}=\partial_{k} f$. The right-hand-side (RHS) is obtained from taking the functional derivative to the RHS of the Wetterich equation 13 and then evaluating at $\psi_{a, 1}(q)=(2 \pi)^{d+1}\left(2 \rho_{0}\right)^{1 / 2} \delta(q), \psi_{a, 2}(q)=0$ and $\tilde{\mu}=\mu$. The interaction terms are obtained from the flow of the longitudinal masses (see Eq. 177)

$$
\begin{aligned}
2 \rho_{0} \dot{\lambda}-\left(\lambda+\lambda_{A B}\right) \dot{\rho}_{0} & =\left.\frac{\delta^{2} \dot{\Gamma}}{\delta \psi_{A, 1}^{2}}\right|_{p=0, \rho_{0}, \mu} \\
2 \rho_{0} \dot{\lambda}_{A B} & =\left.\frac{\delta^{2} \dot{\Gamma}}{\delta \psi_{A, 1} \delta \psi_{B, 1}}\right|_{p=0, \rho_{0}, \mu},
\end{aligned}
$$


where the external momentum $p=(\nu, \mathbf{p})$ of the two-point function $\boldsymbol{\Gamma}^{(2)}$ is evaluated at zero. The renormalization factors are obtained from momentum derivatives of the two-point function 47]

$$
\begin{aligned}
\frac{\dot{Z}}{2 m} & =\left.\frac{\partial}{\partial \mathbf{p}^{2}}\left(\frac{\delta^{2} \dot{\Gamma}}{\delta \psi_{A, 2}^{2}}\right)\right|_{p=0, \rho_{0}, \mu}, \\
\dot{S} & =\left.\frac{\partial}{\partial \nu}\left(\frac{\delta^{2} \dot{\Gamma}}{\delta \psi_{A, 2} \delta \psi_{A, 1}}\right)\right|_{p=0, \rho_{0}, \mu}, \\
\dot{V} & =\left.\frac{\partial}{\partial \nu^{2}}\left(\frac{\delta^{2} \dot{\Gamma}}{\delta \psi_{A, 2}^{2}}\right)\right|_{p=0, \rho_{0}, \mu} .
\end{aligned}
$$

Finally, the flow equations for $n_{0}$ and $n_{1}$ are obtained from taking derivatives with respect to $\mu$ [47]

$$
\begin{aligned}
\dot{n}_{0}-n_{1} \dot{\rho}_{0} & =-\frac{\partial}{\partial \mu} \dot{\Gamma}, \\
\sqrt{2 \rho_{0}} \dot{n}_{1} & =-\frac{\partial}{\partial \mu}\left(\frac{\delta \dot{\Gamma}}{\delta \psi_{A, 1}}\right) .
\end{aligned}
$$

For details on the expressions for the RHS's of the flow equations we refer to Ref. 63].

\section{Appendix B: Dispersion relations and microscopic sound velocity}

From the inverse propagator 17 and by performing a continuation to real time $\omega \rightarrow-i q_{0}$, we can identify the poles of the propagator from $\operatorname{det}\left(\mathbf{G}_{k}^{-1}\right)=0$. Within our truncation, the propagator has eight poles, which can be summarized as

$$
\begin{aligned}
& \left(q_{0,1}^{*}\right)^{2}=\frac{1}{2 V^{2}}\left[S^{2}+V\left(E_{1, k}^{(+)}(\mathbf{q})+E_{2, k}(\mathbf{q})\right)-\sqrt{\left(S^{2}+V\left(E_{1, k}^{(+)}(\mathbf{q})+E_{2, k}(\mathbf{q})\right)\right)^{2}-4 V^{2} E_{1, k}^{(+)}(\mathbf{q}) E_{2, k}(\mathbf{q})}\right], \\
& \left(q_{0,2}^{*}\right)^{2}=\frac{1}{2 V^{2}}\left[S^{2}+V\left(E_{1, k}^{(-)}(\mathbf{q})+E_{2, k}(\mathbf{q})\right)-\sqrt{\left(S^{2}+V\left(E_{1, k}^{(-)}(\mathbf{q})+E_{2, k}(\mathbf{q})\right)\right)^{2}-4 V^{2} E_{1, k}^{(-)}(\mathbf{q}) E_{2, k}(\mathbf{q})}\right] \\
& \left(q_{0,3}^{*}\right)^{2}=\frac{1}{2 V^{2}}\left[S^{2}+V\left(E_{1, k}^{(+)}(\mathbf{q})+E_{2, k}(\mathbf{q})\right)+\sqrt{\left(S^{2}+V\left(E_{1, k}^{(+)}(\mathbf{q})+E_{2, k}(\mathbf{q})\right)\right)^{2}-4 V^{2} E_{1, k}^{(+)}(\mathbf{q}) E_{2, k}(\mathbf{q})}\right] \\
& \left(q_{0,4}^{*}\right)^{2}=\frac{1}{2 V^{2}}\left[S^{2}+V\left(E_{1, k}^{(-)}(\mathbf{q})+E_{2, k}(\mathbf{q})\right)+\sqrt{\left(S^{2}+V\left(E_{1, k}^{(-)}(\mathbf{q})+E_{2, k}(\mathbf{q})\right)\right)^{2}-4 V^{2} E_{1, k}^{(-)}(\mathbf{q}) E_{2, k}(\mathbf{q})}\right],
\end{aligned}
$$

where $E_{1 . k}^{(+)}$is defined in Eq. 27 and $E_{2, k}$ in Eq. 21.

In the following, we examine the behavior of the poles in the different regimes, complementing the discussion in subsection IIIC Later, we examine the microscopic sound velocity extracted from the dispersion relation.

\section{Scale regimes}

For high scales $k \gg k_{h, \pm}$, because $E_{1, k}^{( \pm)} \approx E_{2, k}$ the density and spin modes are indistinguishable. Furthermore, because at these high scales the renormalization factors remain at their microscopic values $Z, S \approx 1$ and $V \approx 0$ (see App. C), the propagator has a single pole

$$
\left(q_{0}^{*}\right)^{2}=E_{2, k}^{2}(\mathbf{q})
$$

which recovers the quadratic spectrum at high momentum as in Bogoliubov theory [2].

For intermediate scales $k_{h,-} \lesssim k \lesssim k_{h,-}$, it is easy to see that the inter-species interaction term $\lambda_{A B}$ becomes important. If we neglect $V$ at these scales, we get the poles

$$
\begin{aligned}
& \left(q_{0,+}^{*}\right)^{2}=E_{1, k}^{(+)}(\mathbf{q}) E_{2, k}(\mathbf{q}) / S^{2}, \\
& \left(q_{0,-}^{*}\right)^{2}=E_{1, k}^{(-)}(\mathbf{q}) E_{2, k}(\mathbf{q}) / S^{2},
\end{aligned}
$$

analogous to those in perturbative approaches [37. In particular, if $k_{h,-} \ll k_{h,+}$, around $k_{h,+}$ we have a quadratic spectrum for the spin mode $\left(q_{0,-}^{*}\right)^{2} \approx$ $E_{2, k}^{2}(\mathbf{q}) / S^{2}$, whereas around $k_{h,-}$ we have a linear spectrum for the density mode $\left(q_{0,+}^{*}\right)^{2} \approx 2 \rho_{0}(\lambda+$ $\left.\lambda_{A B}\right) E_{2, k}(\mathbf{q}) / S^{2}$.

For small scales $k \ll k_{h, \pm}$, the mass terms dominate $E_{1, k}^{(+)}$and the spectrum becomes linear. Particularly insightful is to examine the physical limit $k \rightarrow 0$ of the spectrum in terms of the sound velocity. We do this in the following. 


\section{Microscopic sound velocity}

For $k \rightarrow 0$ the spectrum becomes linear with two microscopic sound velocities $\left(q_{0, \pm}^{*}\right)^{2}=c_{ \pm} \mathbf{q}^{2}$ [36]. From the poles of the sound modes [47], Eqs. (B1) and (B2), we obtain

$$
c_{ \pm}^{2}=\left(\frac{Z / 2 m}{V+S^{2} /\left(2\left(\lambda \pm \lambda_{A B}\right) \rho_{0}\right)}\right)_{k \rightarrow 0},
$$

which for $\lambda_{A B}=0$ takes the known form for a onecomponent gas [45]. Because $\lambda_{A B}, \lambda \rightarrow 0$, both sound velocities are equal $c=c_{ \pm}$, in contrast to the Bogoliubov spectrum that have distinct density and spin sound velocities. Furthermore, because both $S$ and $\lambda$ vanish with the same scaling behavior [45] (see also App. C), we obtain that

$$
c^{2}=\left(\frac{Z}{2 m V}\right)_{k \rightarrow 0}
$$

as with one-component gases [54].

First, we stress that the spectrum remains linear even though the sound velocities differ from that of Bogoliubov theory. Second, as discussed in the main text, the fact that we do not obtain separate density and spin sound velocities results from the form of the effective action at long distances. Density and spin fluctuations are correctly incorporated into the macroscopic properties even though the theory at long distances becomes independent of $\lambda_{A B}$.

It is also relevant to examine if the sound velocity depends on the inter-species interaction. In Fig. 5 we show $c^{2}$ in two and three dimensions for a range of $a_{A B} / a$ for a chosen chemical potential. We obtain that the sound velocity does depend on $a_{A B}$. Moreover, it vanishes for $a_{A B} \rightarrow a$, signaling the phase separation point. It is interesting that, since the interaction couplings vanish, the decrease of $c^{2}$ is driven by the one-component couplings $Z$ and $V$.

Here we note that in a Bogoliubov formulation, the spin sound velocity vanishes at the phase separation point, whereas the density sound velocity remains finite 36 . There is no apparent reason beforehand why the FRG flow gives a vanishing microscopic sound velocity at the phase separation. A detailed analysis of the critical point is required, which is left to future work.

\section{Appendix C: FRG flows}

As mentioned in subsection IV A, the flows of the mixture gas are similar to those of one-component gases (see Refs. 45 47 for details). Here we examine the flows of the different $k$-dependent functions. We compare the flows with calculations using $a_{A B}=0$. In this limit, $\lambda_{A B}=0$ for all $k$ and the flow equations recover their forms for one-component gases.

Fig. 6 shows flows of the renormalization factors $Z, S$ and $V$. The inter-species interaction produces a deviation of all the couplings from their flows for $a_{A B}=0$. However, the couplings keep their infrared behavior, as discussed in the main text. Also, note that the flows deviate from each other as $k$ approaches $k_{h,+}$, showing that the inter-species interaction is not important at higher scales.

The mass renormalization $Z$ increases from its microscopic value at high scales to converge to a constant for $k \rightarrow 0$, whereas the wave-function renormalization $S$ vanishes in the IR as $k$ in two dimensions and logarithmically in three dimensions. The coupling $V$, which is not present in the microscopic action (1), is generated by the quantum fluctuations as we lower $k$. As examined in detail in Ref. 46, the long distance behavior of the propagator is dominated by the quadratic frequency term $V$ instead of the linear term $S$. Thus, the IR is described by a relativistic-like model. In two dimensions, it is easy to see that $V$ dominates the IR physics, as $V$ quickly converges to finite values for $k<k_{h,-}$, whereas $S$ vanishes. This shows the importance of fluctuations in two dimensions, as the long-distance theory deviates considerably from the Bogoliubov picture. On the other hand, in three dimensions, the logarithmic flows mean that the coupling $V$ is important only in the extreme IR. Its inclusion does not considerably affect the macroscopic properties. Still, $V$ should converge to a finite value at $k=0$, and its inclusion is important to obtain a consistent theory.

Fig. 7 shows the flows of the interaction couplings $\lambda$ and $\lambda_{A B}$. The intra-species coupling $\lambda$ is insensitive to the inter-species interaction, with indistinguishable flows for different values of $a_{A B}$. In contrast, $\lambda_{A B}$ depends strongly on $a_{A B}$, as expected. As discussed in the main text, both $\lambda$ and $\lambda_{A B}$ vanish for $k \rightarrow 0$, with $\lambda$ vanishing as $k$ in two dimensions and logarithmically in three dimensions. Consistent with our analysis for the ratio $\lambda_{A B} / \lambda, \lambda_{A B}$ vanishes as $k^{2}$ in two dimensions and logarithmically in three dimensions.

Finally, Fig. 8 shows flows of the condensate density $\rho_{0}$. These are rescaled in terms of mean-field values for the one-component gas. In all cases, $\rho_{0}$ converges to finite values for $k \rightarrow 0$, consistent with a finite condensate density.
[1] C. J. Pethick and H. Smith, Bose-Einstein Condensation in Dilute Gases, 2nd ed. (Cambridge University Press,
Cambridge, 2008). 


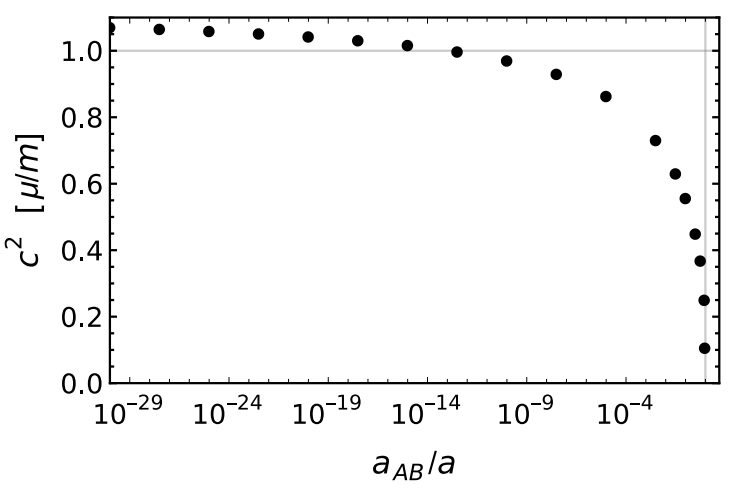

(a) Two dimensions

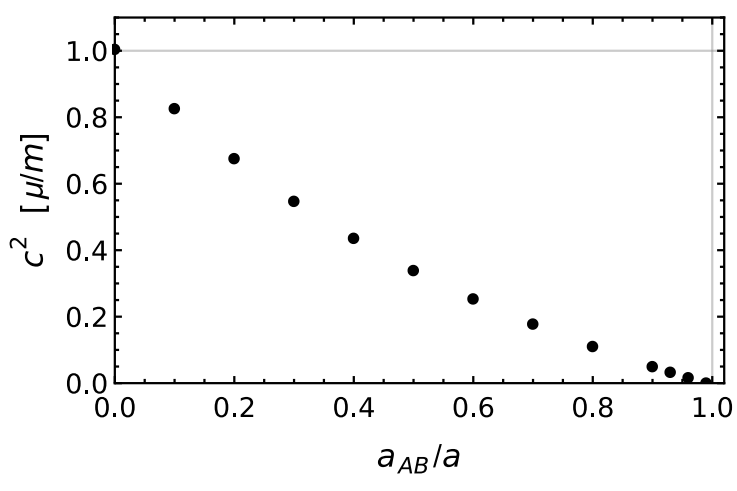

(b) Three dimensions

FIG. 5. Sound velocity $c^{2}$ obtained from FRG calculations as a function of $a_{A B} / a$ for $m a^{2} \mu=10^{-4}$.
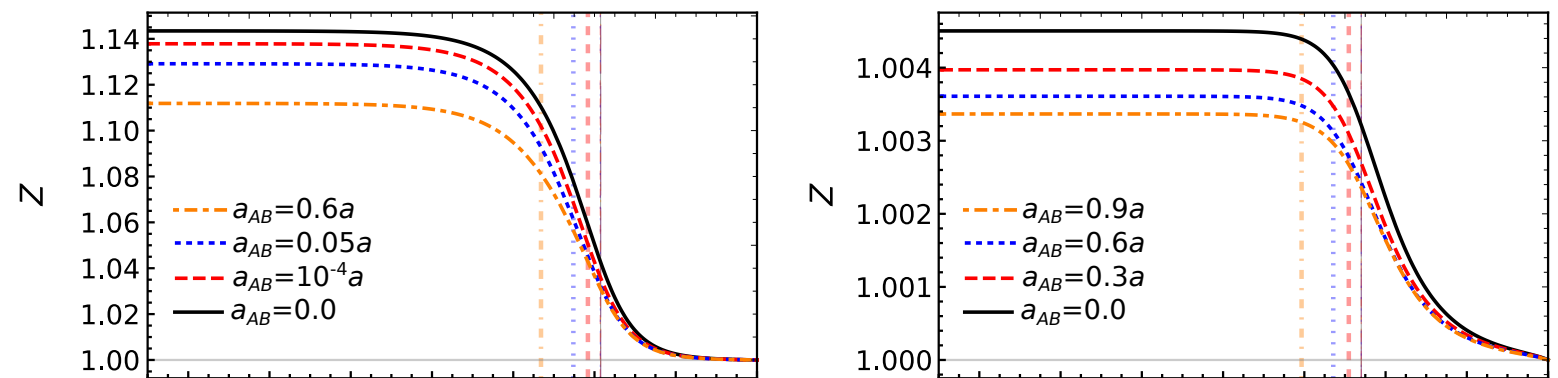

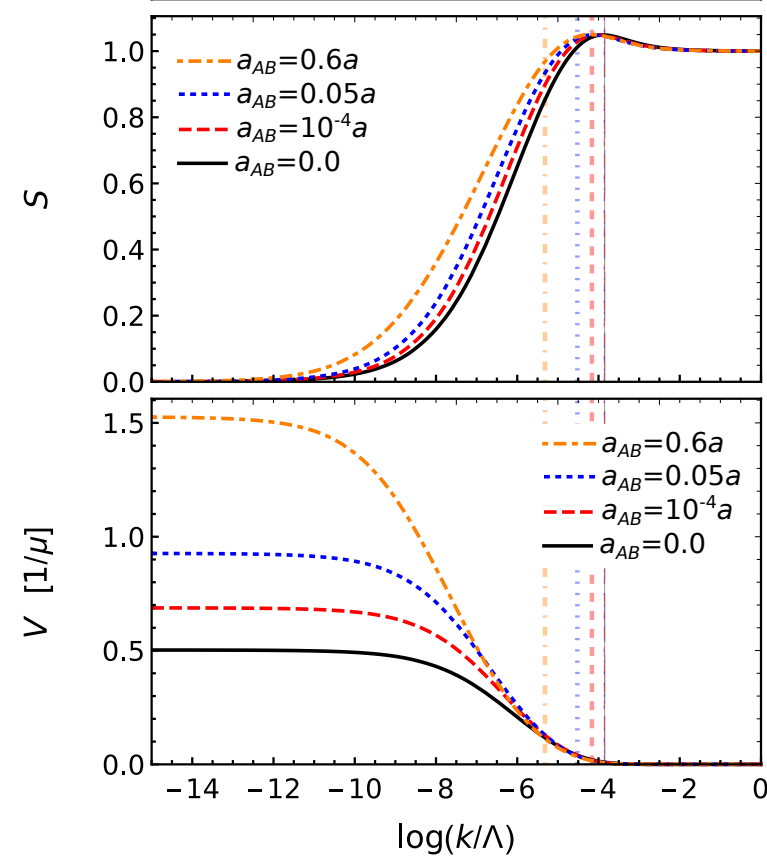

(a) Two dimensions
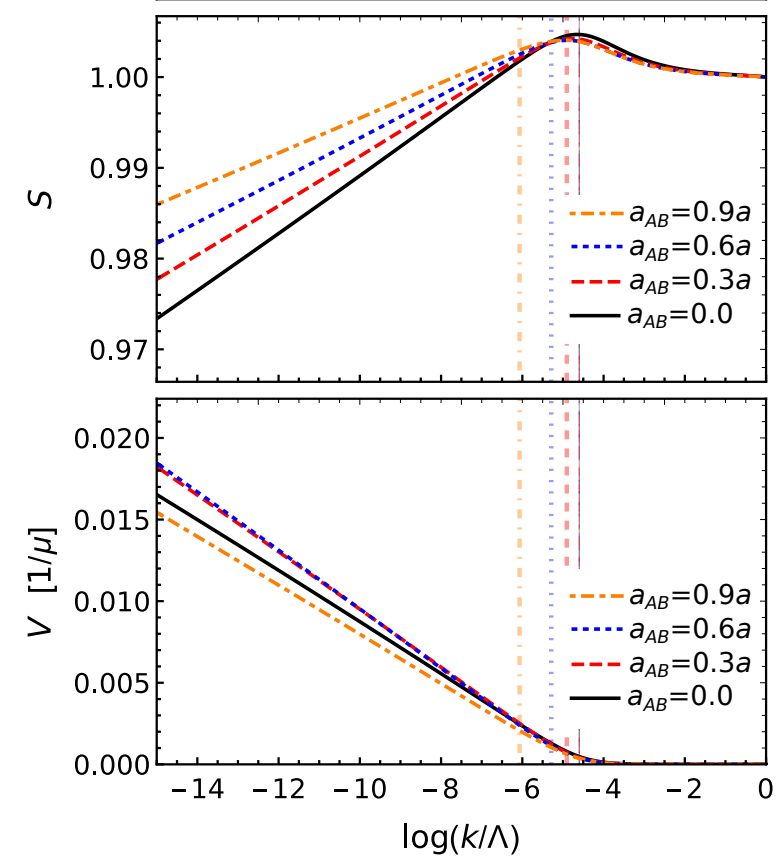

(b) Three dimensions

FIG. 6. Flows of $Z, S$ and $V$ as a function of $k$ in two and three dimensions for $m a^{2} \mu=10^{-4}$. Thin and thick vertical lines denote $k_{h,+}$ and $k_{h,-}$, respectively.

[2] L. P. Pitaevskii and S. Stringari, Bose-Einstein condensation and superfluidity (Oxford University Press, Oxford, 2016).
[3] M. H. Anderson, J. R. Ensher, M. R. Matthews, C. E. Wieman, and E. A. Cornell, Observation of Bose-Einstein Condensation in a Dilute Atomic Vapor, Science 269, 198 


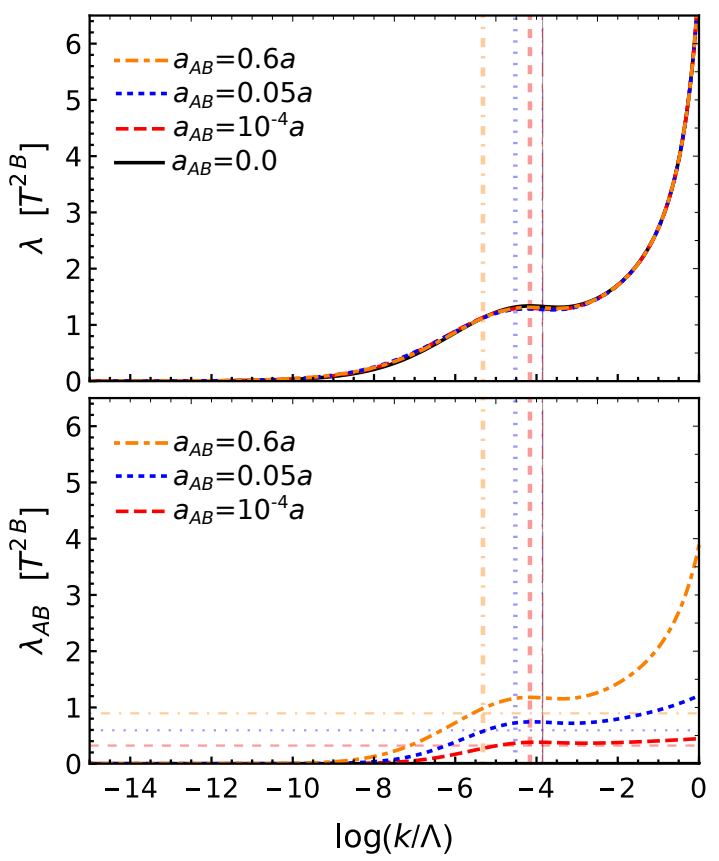

(a) Two dimensions

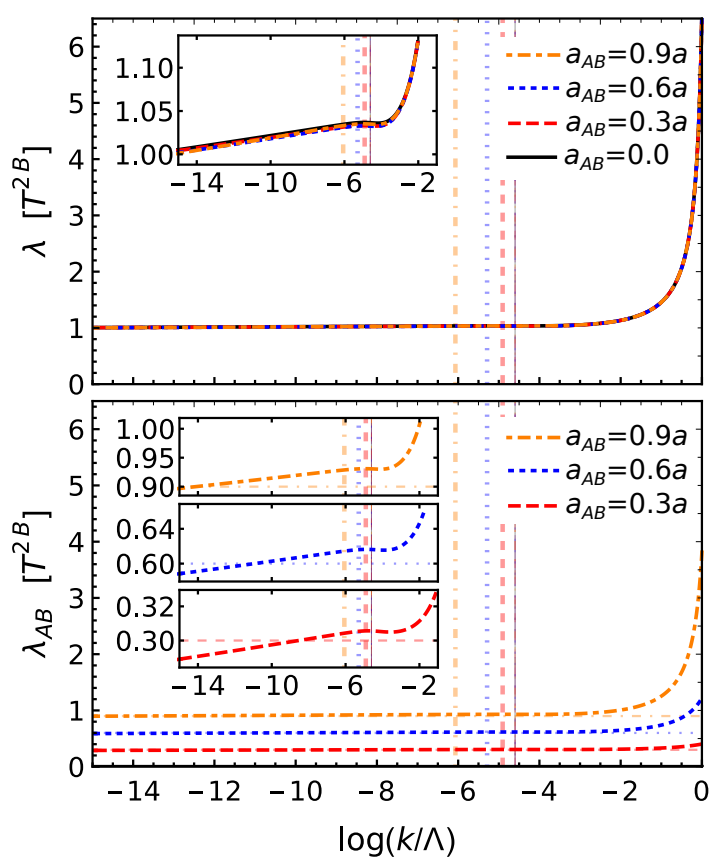

(b) Three dimensions

FIG. 7. Flows of $\lambda$ and $\lambda \overline{A B}$ as a function of $k$ in two and three dimensions for $m a^{2} \mu=10^{-4}$. Both couplings are rescaled in terms of the intra-species $T$-matrices defined in Eq. (24). Thin and thick vertical lines denote $k_{h,+}$ and $k_{h,-}$, respectively. (Bottom) Solid horizontal lines correspond to the ratios $T^{\mathbb{Z} \mathrm{B}} / T_{A B}^{2 \mathrm{~B}}$.

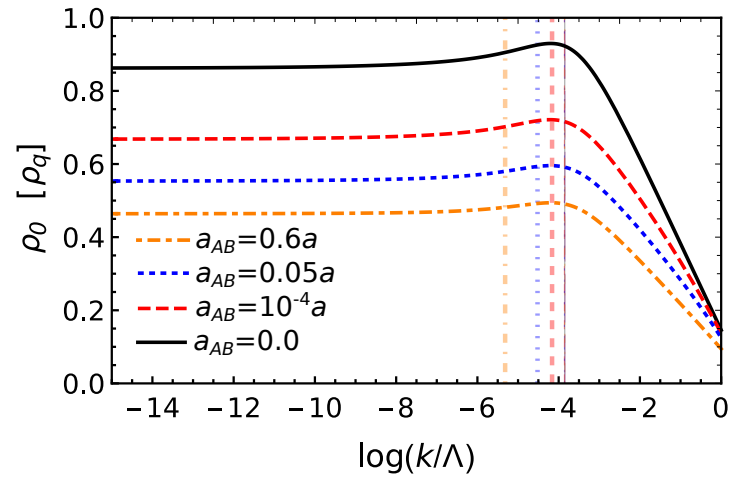

(a) Two dimensions

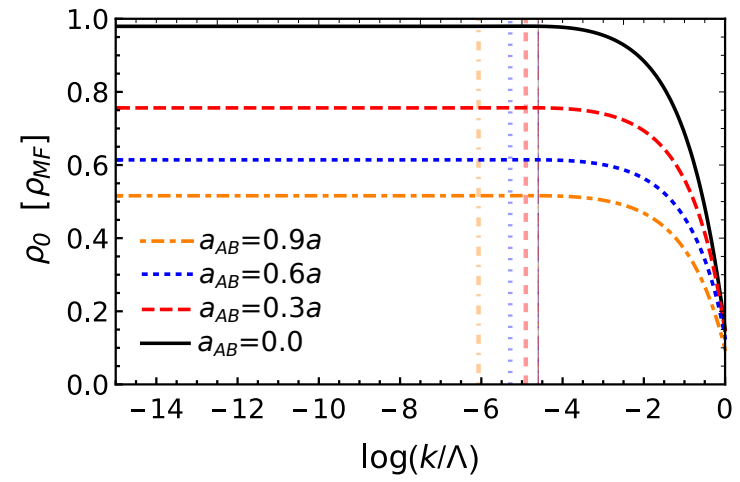

(b) Three dimensions

FIG. 8. Flows of $\rho_{0}$ as a function of $k$ in two and three dimensions for $m a^{2} \mu=10$. In three dimensions $\rho_{00}$ is rescaled in terms of its MF value for a one-component gas $\overline{A \mathrm{MF}}=\mu m / 4 \pi a$. Similarly, in two dimensions $\rho_{0}$ is rescaled in terms of $f_{q q}=\mu m \log \left(4 / \mu m a^{2, d^{2 \gamma_{E}+1}}\right) / 4 \pi[78]$. Thin and thick vertical lines denote $k_{h,+}$ and $k_{[,-,}$respectively.

\section{(1995)}

[4] K. B. Davis, M. O. Mewes, M. R. Andrews, N. J. van Druten, D. S. Durfee, D. M. Kurn, and W. Ketterle, Bose-Einstein Condensation in a Gas of Sodium Atoms, Physical Review Letters 75, 3969 (1995)

[5] C. C. Bradley, C. A. Sackett, J. J. Tollett, and R. G. Hulet, Evidence of Bose-Einstein Condensation in an Atomic Gas with Attractive Interactions, Physical Review Letters 75, 1687 (1995).

[6] I. Bloch, J. Dalibard, and W. Zwerger, Many-body physics with ultracold gases, Reviews of Modern Physics $\mathbf{8 0 ,} 885(2008)$

[7] N. Bogoliubov, On the theory of superfluidity, Izv. AN SSSR Ser. Fiz. 11, 77 (1947).

[8] T. D. Lee and C. N. Yang, Many-Body Problem in Quantum Mechanics and Quantum Statistical Mechanics, Physical Review 105, 1119 (1957).

[9] T. D. Lee, K. Huang, and C. N. Yang, Eigenvalues and Eigenfunctions of a Bose System of Hard Spheres and Its Low-Temperature Properties, Physical Review 106, 1135 (1957)

[10] J. Andersen, Theory of the weakly interacting Bose gas, 
Reviews of Modern Physics 76, 599 (2004).

[11] U. Al Khawaja, J. O. Andersen, N. P. Proukakis, and H. T. C. Stoof, Low dimensional Bose gases, Physical Review A 66, 013615 (2002).

[12] A. Posazhennikova, Colloquium : Weakly interacting, dilute Bose gases in 2D, Reviews of Modern Physics 78, $1111(2006)$

[13] Z. Hadzibabic and J. Dalibard, Two-dimensional Bose fluids: An atomic physics perspective, La Rivista del Nuovo Cimento 34, 389 (2011)

[14] M. A. Cazalilla, R. Citro, T. Giamarchi, E. Orignac, and M. Rigol, One dimensional bosons: From condensed matter systems to ultracold gases, Reviews of Modern Physics 83, 1405 (2011)

[15] A. F. Andreev and E. P. Bashkin, Three-velocity hydrodynamics of superfluid solutions, Sov. Phys. JETP 42, 164 (1975).

[16] D. V. Fil and S. I. Shevchenko, Nondissipative drag of superflow in a two-component Bose gas, Physical Review A 72, 013616 (2005)

[17] J. Nespolo, G. E. Astrakharchik, and A. Recati, Andreev-Bashkin effect in superfluid cold gases mixtures, New Journal of Physics 19, 125005 (2017).

[18] P. Tommasini, E. J. V. de Passos, A. F. R. de Toledo Piza, M. S. Hussein, and E. Timmermans, Bogoliubov theory for mutually coherent condensates, Physical Review A 67, 023606 (2003).

[19] M. Abad and A. Recati, A study of coherently coupled two-component Bose-Einstein condensates, The European Physical Journal D 67, 148 (2013).

[20] P. Ao and S. T. Chui, Binary Bose-Einstein condensate mixtures in weakly and strongly segregated phases, Physical Review A 58, 4836 (1998).

[21] E. Timmermans, Phase Separation of Bose-Einstein Condensates, Physical Review Letters 81, 5718 (1998)

[22] M. Trippenbach, K. Góral, K. Rzazewski, B. Malomed, and Y. B. Band, Structure of binary Bose-Einstein condensates, Journal of Physics B: Atomic, Molecular and Optical Physics 33, 4017 (2000).

23] D. Petrov, Quantum Mechanical Stabilization of a Collapsing Bose-Bose Mixture, Physical Review Letters 115, 155302 (2015)

[24] D. Petrov and G. Astrakharchik, Ultradilute LowDimensional Liquids, Physical Review Letters 117, 100401 (2016)

[25] T.-L. Ho and V. B. Shenoy, Binary Mixtures of Bose Condensates of Alkali Atoms, Physical Review Letters $\mathbf{7 7}, 3276(1996)$.

[26] C. J. Myatt, E. A. Burt, R. W. Ghrist, E. A. Cornell, and C. E. Wieman, Production of Two Overlapping BoseEinstein Condensates by Sympathetic Cooling, Physical Review Letters 78, 586 (1997)

[27] D. S. Hall, M. R. Matthews, J. R. Ensher, C. E. Wieman, and E. A. Cornell, Dynamics of Component Separation in a Binary Mixture of Bose-Einstein Condensates, Physical Review Letters 81, 1539 (1998)

[28] D. S. Hall, M. R. Matthews, C. E. Wieman, and E. A. Cornell, Measurements of Relative Phase in TwoComponent Bose-Einstein Condensates, Physical Review Letters 81, 1543 (1998)

[29] G. Modugno, M. Modugno, F. Riboli, G. Roati, and M. Inguscio, Two Atomic Species Superfluid, Physical Review Letters 89, 190404 (2002).

[30] L. Chomaz, S. Baier, D. Petter, M. Mark, F. Wächtler,
L. Santos, and F. Ferlaino, Quantum-Fluctuation-Driven Crossover from a Dilute Bose-Einstein Condensate to a Macrodroplet in a Dipolar Quantum Fluid, Physical Review X 6, 041039 (2016).

[31] I. Ferrier-Barbut, H. Kadau, M. Schmitt, M. Wenzel, and T. Pfau, Observation of Quantum Droplets in a Strongly Dipolar Bose Gas, Physical Review Letters 116, 215301 (2016)

[32] A. Cappellaro, T. Macrì, G. F. Bertacco, and L. Salasnich, Equation of state and self-bound droplet in Rabicoupled Bose mixtures, Scientific Reports 7, 13358 (2017)

[33] C. R. Cabrera, L. Tanzi, J. Sanz, B. Naylor, P. Thomas, P. Cheiney, and L. Tarruell, Quantum liquid droplets in a mixture of Bose-Einstein condensates, Science 359, 301 (2018)

[34] D. M. Larsen, Binary mixtures of dilute bose gases with repulsive interactions at low temperature, Annals of Physics 24, 89 (1963).

[35] B. Oleś and K. Sacha, $N$-conserving Bogoliubov vacuum of a two-component Bose-Einstein condensate: density fluctuations close to a phase-separation condition, Journal of Physics A: Mathematical and Theoretical 41, 145005 (2008).

[36] J. Armaitis, H. T. C. Stoof, and R. A. Duine, Hydrodynamic modes of partially condensed Bose mixtures, Physical Review A 91, 043641 (2015).

[37] E. Chiquillo, Equation of state of the one- and threedimensional Bose-Bose gases, Physical Review A 97, 063605 (2018).

[38] P. Konietin and V. Pastukhov, 2D Dilute Bose Mixture at Low Temperatures, Journal of Low Temperature Physics 190, 256 (2018)

[39] M. Ota and G. E. Astrakharchik, Beyond LeeHuang-Yang description of self-bound Bose mixtures, arXiv:2005.10047 [cond-mat] (2020), arXiv: 2005.10047.

[40] O. I. Utesov, M. I. Baglay, and S. V. Andreev, Effective interactions in a quantum Bose-Bose mixture, Physical Review A 97, 053617 (2018)

[41] L. Parisi, G. Astrakharchik, and S. Giorgini, Liquid State of One-Dimensional Bose Mixtures: A Quantum Monte Carlo Study, Physical Review Letters 122, 105302 (2019)

[42] V. Cikojević, L. V. Markić, G. E. Astrakharchik, and J. Boronat, Universality in ultradilute liquid Bose-Bose mixtures, Physical Review A 99, 023618 (2019).

[43] C. Wetterich, Exact evolution equation for the effective potential, Physics Letters B 301, 90 (1993).

[44] J. Berges, N. Tetradis, and C. Wetterich, Nonperturbative renormalization flow in quantum field theory and statistical physics, Physics Reports 363, 223 (2002)

[45] N. Dupuis and K. Sengupta, Non-perturbative renormalization group approach to zero-temperature Bose systems, Europhysics Letters 80, 50007 (2007)

[46] C. Wetterich, Functional renormalization for quantum phase transitions with nonrelativistic bosons, Physical Review B 77, 064504 (2008)

[47] S. Floerchinger and C. Wetterich, Functional renormalization for Bose-Einstein condensation, Physical Review A 77, 053603 (2008)

[48] S. Floerchinger and C. Wetterich, Superfluid Bose gas in two dimensions, Physical Review A 79, 013601 (2009)

[49] S. Floerchinger and C. Wetterich, Nonperturbative ther- 
modynamics of an interacting Bose gas, Physical Review A 79, 063602 (2009)

[50] N. Dupuis, Infrared behavior and spectral function of a Bose superfluid at zero temperature, Physical Review A 80, 043627 (2009).

[51] A. Sinner, N. Hasselmann, and P. Kopietz, Functional renormalization-group approach to interacting bosons at zero temperature, Physical Review A 82, 063632 (2010).

[52] S. Lammers, I. Boettcher, and C. Wetterich, Dimensional crossover of nonrelativistic bosons, Physical Review A 93, 063631 (2016).

[53] F. Isaule, M. C. Birse, and N. R. Walet, Thermodynamics of Bose gases from functional renormalization with a hydrodynamic low-energy effective action, Annals of Physics 412, 168006 (2020).

[54] N. Dupuis, Infrared behavior in systems with a broken continuous symmetry: Classical $\mathrm{O}(N)$ model versus interacting bosons, Physical Review E 83, 031120 (2011).

[55] J. Gavoret and P. Nozières, Structure of the perturbation expansion for the bose liquid at zero temperature, Annals of Physics 28, 349 (1964).

[56] A. A. Nepomnyashchii and Y. A. Nepomnyashchii, Contribution to the theory of the spectrum of a Bose system with condensate at small momenta, Pis'ma Zh. Eksp. Teor. Fiz. 21, 3 (1975).

[57] A. Rançon and N. Dupuis, Nonperturbative renormalization group approach to the Bose-Hubbard model, Physical Review B 83, 172501 (2011).

[58] A. K. Kolezhuk, Stability of low-dimensional multicomponent dilute Bose gases, Physical Review A 81, 013601 (2010)

[59] Y.-L. Lee and Y.-W. Lee, The Stability of the LowDimensional Mixtures of Dilute Quantum Gases, Journal of the Physical Society of Japan 80, 044003 (2011)

[60] G. Ceccarelli, J. Nespolo, A. Pelissetto, and E. Vicari, Bose-Einstein condensation and critical behavior of twocomponent bosonic gases, Physical Review A 92, 043613 (2015)

[61] V. Karle, N. Defenu, and T. Enss, Coupled superfluidity of binary Bose mixtures in two dimensions, Physical Review A 99, 063627 (2019).

[62] H. T. C. Stoof, K. B. Gubbels, and D. Dickerscheid, Ultracold Quantum Fields (Springer, Dordrecht, 2009).

[63] B. Delamotte, D. Mouhanna, and M. Tissier, Nonperturbative renormalization-group approach to frustrated magnets, Physical Review B 69, 134413 (2004)

[64] P. Kopietz, L. Bartosch, and F. Schüt, Introduction to the Functional Renormalization Group (Springer, Heidelberg, 2010).

[65] I. Boettcher, J. M. Pawlowski, and S. Diehl, Ultra- cold atoms and the Functional Renormalization Group, Nuclear Physics B - Proceedings Supplements 228, 63 (2012)

[66] N. Dupuis, L. Canet, A. Eichhorn, W. Metzner, J. M. Pawlowski, M. Tissier, and N. Wschebor, The nonperturbative functional renormalization group and its applications, arXiv:2006.04853 [cond-mat, physics:gr-qc, physics:hep-ph, physics:hep-th] (2020).

[67] V. N. Popov, Functional integrals and collective excitations (Cambridge University Press, Cambridge, 1987).

[68] D. F. Litim, Optimisation of the exact renormalisation group, Physics Letters B 486, 92 (2000).

[69] J. O. Andersen, U. Al Khawaja, and H. T. C. Stoof, Phase Fluctuations in Atomic Bose Gases, Physical Review Letters 88, 070407 (2002).

[70] L. Parisi, G. Astrakharchik, and S. Giorgini, Spin Dynamics and Andreev-Bashkin Effect in Mixtures of OneDimensional Bose Gases, Physical Review Letters 121, 025302 (2018)

71] M. Ota and S. Giorgini, Thermodynamics of dilute Bose gases: Beyond mean-field theory for binary mixtures of Bose-Einstein condensate, arXiv:2008.05246 [cond-mat] (2020), arXiv: 2008.05246.

[72] R. Lopes, C. Eigen, N. Navon, D. Clément, R. P. Smith, and Z. Hadzibabic, Quantum Depletion of a Homogeneous Bose-Einstein Condensate, Physical Review Letters 119, 190404 (2017).

[73] G. E. Astrakharchik, J. Boronat, J. Casulleras, I. L. Kurbakov, and Y. E. Lozovik, Equation of state of a weakly interacting two-dimensional Bose gas studied at zero temperature by means of quantum Monte Carlo methods, Physical Review A 79, 051602 (2009).

[74] V. Pastukhov, Ground-State Properties of a Dilute TwoDimensional Bose Gas, Journal of Low Temperature Physics 194, 197 (2019).

[75] N. D. Mermin and H. Wagner, Absence of Ferromagnetism or Antiferromagnetism in One- or TwoDimensional Isotropic Heisenberg Models, Physical Review Letters 17, 1133 (1966).

[76] H. Hu and X.-J. Liu, Microscopic derivation of the extended Gross-Pitaevskii equation for quantum droplets in binary Bose mixtures, Physical Review A 102, 043302 (2020).

[77] H. Hu, J. Wang, and X.-J. Liu, Microscopic pairing theory of a binary Bose mixture with interspecies attractions: Bosonic BEC-BCS crossover and ultradilute lowdimensional quantum droplets, Physical Review A 102, $043301(2020)$

[78] C. Mora and Y. Castin, Extension of Bogoliubov theory to quasicondensates, Physical Review A 67, 053615 (2003). 